\title{
MedienPädagogik
}

Zeitschrift für Theorie und Praxis der Medienbildung

\section{Tablets an Beruflichen Gymnasien: Gelingensfaktoren für die Integration mobiler Endgeräte im Schulunterricht} Ausgewählte Ergebnisse des Projekts «tabletBS» 20152019

\author{
Kerstin Mayrberger und Kathrin Galley
}

\section{Zusammenfassung}

Der vorliegende Beitrag stellt ausgewählte Ergebnisse aus dem seit 2015 laufenden Projekt «tabletBS - Einsatz von Tablets an Beruflichen Schulen» vor. Im Rahmen des Projekts «tabletBS» werden ausgewählte Klassen an Beruflichen Gymnasien mit unterschiedlichen Profilen in Baden-Württemberg 1:1 mit Tablets ausgestattet. Evaluiert werden insgesamt rund 144 Klassen aus 40 Schulen in drei Tranchen à ca. 12 Schulen pro Jahr, die wiederum über die dreijährige Zeit der Oberstufe begleitet werden. Das Evaluationsdesign basiert vor allem auf quantitativen Erhebungsmethoden in Form von Online-Fragebögen zur Erfassung aller beteiligten Perspektiven, die nach Bedarf mit qualitativen Erhebungsmethoden für ausgewählte Perspektiven und für vertiefende Fragestellungen ergänzt werden. Es lässt sich nach nunmehr vier Jahren Projektlaufzeit zusammenfassend festhalten, dass Tablets einen tendenziell positiven Einfluss auf die schülerorientierte Entwicklung der Unterrichtsgestaltung haben und dass sie eine gute Beziehungsgestaltung zwischen Lehrenden und Lernenden fördern können. Zudem konnte festgestellt werden, dass eine zunehmende Akzeptanz und Verstetigung der Tabletintegration im Unterricht stattfindet. Wichtig sind hierbei engagier- 
te Lehrende, die das Projektvorhaben in den Schulen wesentlich mitentwickeln, unterstützende Schulleitungen sowie passende Austausch- und Qualifizierungsformate.

\title{
Tablets at vocational grammar-schools: success factors for the integration of mobile end devices in school teaching. Selected results of the «tabletBS» project 2015-2019
}

\begin{abstract}
This article presents selected results from the project «tabletBS - Use of tablets at vocational schools》 which has been running since 2015. Within the project «tabletBS» selected classes at vocational high schools with different profiles in Baden-Württemberg are equipped 1:1 with tablets. A total of around 144 classes from 40 schools will be evaluated in three tranches of approximately 12 schools per year, which in turn will be monitored over the three-year period of the upper school. The evaluation design is based primarily on quantitative survey methods in the form of online questionnaires to record all perspectives involved, which are supplemented as required with qualitative survey methods for selected perspectives and for more in-depth questions. After four years of project duration, it can be summarised that tablets tend to have a positive influence on the student-oriented development of teaching design and that they can promote a good relationship between teachers and students. In addition, it was found that tablet integration is becoming increasingly accepted and established in class. Important factors in this regard are committed teachers who play a major role in developing the project in the schools, supportive school administrations and suitable exchange and qualification formats.
\end{abstract}

\section{Einleitung}

Die Aktualität und Relevanz der Frage nach einer sinnvollen fachlichen und didaktischen Implementierung von mobilen Technologien im schulischen Kontext lässt sich derzeit besonders gut an der Ende 2019 in Deutschland geführten Debatte um die Verwendung der Ressourcen aus 
dem «DigitalPakt Schule» aufzeigen (https://www.digitalpaktschule.de sowie aus empirischer Sicht s. Scheiter und Lachner 2019). Zugleich macht die Veröffentlichung der internationalen IEA-Vergleichsstudie ICILS 2018 (International Computer and Information Literacy Study) deutlich, dass die Digitalisierung der Schulen noch nicht zufriedenstellend ist (Eickelmann et al. 2019) und, wie Birgit Eickelmann als Sprecherin des deutschen ICILSKonsortiums in einem Interview zur Publikation der Studie treffend betont:

«Die ICILS-2018-Studie macht erneut im internationalen Vergleich deutlich, dass viele Entwicklungsaufgaben im Zuge der Digitalisierung im Schulbereich in Deutschland noch mit mehr Nachdruck bearbeitet werden müssen. Eine besondere Zukunftsaufgabe wird sein, alle Schülerinnen und Schüler zu mündigen Bürgerinnen und Bürgern in einer von Digitalisierung geprägten Welt zu befähigen.» (Eickelmann 2019)

Hier wird deutlich, dass neben der Infrastruktur als wichtige aber nicht hinreichende Rahmenbedingung die medienbezogenen Kompetenzen in Form von Praktiken bzw. die Praxis der selbstverständlichen Mediennutzung im schulischen Unterricht noch nicht ausgereift ist. Mit Bezug auf Stalders Kultur der Digitalität (Stalder 2016) könnte man insofern folgern, dass weder die Digitalisierung im Sinne einer Technisierung, noch die Praktiken unter den Bedingungen der Digitalität im Sinne einer Veränderung der Strukturbedingungen des Handelns durch Referenzialität, Gemeinschaftlichkeit und Algorithmizität als drei kulturelle Formen von Digitalität im schulischen Kontext in passender und altersangemessener Weise hinreichend entwickelt sind. Insofern liesse sich in Anlehnung an Stalder $(2018,15)$ mit Blick auf die konkretere Frage nach einem gelingenden Tablet-Einsatz im schulischen Unterricht Folgendes schliessen: Eine infrastrukturbezogene, technologiefokussierte Entscheidung scheint erst zielführend getroffen werden zu können, wenn man sich im Vorfeld verständigt, welche medien- und fachbezogenen didaktischen und pädagogischen Ziele verfolgt werden und damit einhergehend, inwiefern die schulischen Praktiken unter den Bedingungen von Digitalisierung und Digitalität umgestaltet werden sollen (vgl. ebd.). Zugleich bleibt hier - auch mit Blick auf 
Beobachtungen im Zuge von «tabletBS» einzuwenden, dass ohne zumindest basale Erfahrungen in der Erprobung von aktuellen Technologien nicht vorausgesetzt werden kann, dass Lehrende didaktisch sinnvoll einschätzen können, wie sie die derzeitigen Technologien mittelfristig sinnvoll in ihre Unterrichtspraxis integrieren können. Somit macht es keinen Sinn im Zuge von Projekten zur Implementierung von Lehrinnovationen die Technologie gegenüber der Pädagogik zu priorisieren oder umgekehrt. Es erscheint im Sinne einer nachhaltigen und akzeptierten Verankerung neuer medienbezogener Praktiken und Herstellung einer Praxis mobilen Lernens nur sinnvoll, wenn Entscheidungen für eine passende Technologie und passende Pädagogik bzw. Didaktik interdependent getroffen werden und eine passende Lösung iterativ entwickelt wird.

Der vorliegende Beitrag stellt ausgewählte Ergebnisse nach vier Jahren Projektlaufzeit aus dem seit 2015 laufenden Projekt «tabletBS - Einsatz von Tablets an Beruflichen Schulen» vor (vgl. für den ersten Zwischenstand Galley und Mayrberger 2018) und fokussiert dabei auf Gelingensund Hinderungsfaktoren beim Einsatz von Tablets im Schulunterricht. Im Rahmen des Projekts «tabletBS» werden einzelne Klassen an ausgewählten Beruflichen Gymnasien mit unterschiedlichen Schwerpunkten in BadenWürttemberg mit Tablets ausgestattet. Mit dem Fokus auf die mediendidaktische Frage «Auf welche Art und Weise können Tablets zur Gestaltung von Lernprozessen - auch im Sinne der individuellen Förderung - in einzelnen Fächern eingesetzt werden?» wird das Projekt wissenschaftlich evaluiert. Projektbeginn war im September 2015, so dass nun nach knapp vier Jahren Projektlaufzeit Ergebnisse vorliegen, die insbesondere aufzeigen, mit welchen Herausforderungen sich die «digitale Bildung» in den Schulen bei der praktischen Integration im Unterrichtsalltag konfrontiert sieht. Über den Projektverlauf hinweg wurden neben regelmässigen quantitativen Online-Befragungen im Längsschnitt verschiedene punktuelle qualitative Erhebungen mit unterschiedlichen Schwerpunkten durchgeführt. Insbesondere die Befragung von Mitgliedern der Schulleitungen in Einzelinterviews sowie aktuell durchgeführte Schulbesuche vor Ort an ausgewählten Schulen in Kombination mit Gruppendiskussionen mit den dortigen Lehrkräften geben wichtige Einblicke darin, unter welchen Bedingungen eine gelungene Integration von Tablets im Schulunterricht erfolgt kann. Dabei 
zeigt sich zugleich, dass der von Beginn der Studie an fokussierte Aspekt der Individualisierung im schulischen Alltag und Unterricht nicht gesondert zum Tragen kam und daher in den nachfolgenden Ausführungen nicht im Mittelpunkt steht, sondern an geeigneter Stelle mit ausgeführt wird.

\section{Zur fachlichen Einordnung}

Der Einsatz oder die Integration von Tablets im schulischen Unterricht kann auch stellvertretend für die Fragen nach einem sinnvollen Einsatz digitaler Medien im schulischen Unterricht stehen. Aus diesem Grunde soll hier beim Forschungsstand auch zuerst auf übergreifende Erkenntnisse Bezug genommen werden, die auch für das konkrete «tabletBS»-Vorhaben eine rahmende Relevanz haben. So kommt Heike Schaumburg (2018) in einem Überblicksbeitrag zu empirischen Befunden zur Wirksamkeit unterschiedlicher Konzepte des digital unterstützten Lernens zu dem Schluss, dass sich nach über 40 Jahren Forschung zur Lerneffektivität digitaler Medien folgendes festhalten liesse:

1. Digitale Medien verbessern die Lerneffektivität. Schlimmstenfalls sind sie unschädlich.

2. Das lernförderliche Potenzial digitaler Medien wird im Rahmen konstruktivistischer Unterrichtsmethoden eher ausgeschöpft als im Rahmen eines lehrerzentrierten Unterrichts.

3. Auf die Lehrkraft kommt es an - der Einsatz digitaler Medien im Rahmen konstruktivistischer Unterrichtsmethoden muss sorgfältig vorbereitet, begleitet und ausgewertet werden, um das lernförderliche Potenzial auszureizen. (Schaumburg 2018, 38)

Diese Folgerungen zeigen auf, dass eine technikzentrierte Perspektive, d.h. die Frage, welche Informations- und Kommunikationstechnologien am Ende angeschafft und genutzt werden oder welche Software welcher Firma es wird, für einen gelingenden Unterricht nicht förderlich ist. Für den vorliegenden Kontext ist dabei besonders relevant, dass «die Art und Weise der didaktischen Einbindung in den Unterricht entscheidend für die Lerneffektivität digitaler Medien ist und dass schülerorientierte und konstruktivistische Ansätze hier ein grösseres Potenzial aufweisen als die 
Einbindung in einen lehrerzentrierten Unterricht» (Schaumburg 2018, 37). Und folglich stellt sich dann eine Frage nach den medienbezogenen Praktiken, die die Schülerinnen und Schüler im Zuge einer Unterrichtspraxis unter den Bedingungen von Digitalisierung und Digitalität erfahren, erleben und inkorporieren können. Denn weiter zeige sich, «dass das lernförderliche Potenzial digitaler Medien im Rahmen schülerorientierter, problemorientierter und offener Unterrichtsformen offenbar besser erschlossen werden kann als in einem lehrerzentrierten Unterricht. Die vorliegenden Meta-Analysen geben Hinweise, dass im Kontext solcher auch als „konstruktivistisch" bezeichneten methodischen Unterrichtsarrangements eine (teilweise bedeutend) höhere Lerneffektivität erzielt wird» (Schaumburg 2018, 36-37). Die Entscheidung für bestimmte mediendidaktische Praktiken im Fachunterricht oder weiter gesprochen medienpädagogische Handlungspraxen in der Schule ist hier also von grosser Bedeutung.

Konkret bezogen auf den Einsatz von Tablets im Unterricht, die hier als zeitgemässe Variante der eben beschriebenen digitalen Medien im schulischen Unterricht betrachtet werden, liegen einige Evaluationsstudien und konzeptionelle Überlegungen für und aus dem schulischen Kontext zur Weiterentwicklung von Lehr-Lernumgebungen mit mobilen Endgeräten vor (u.a. Bastian und Aufenanger 2017; Heinz 2018; Mayrberger 2018; Gerick und Eickelmann 2018; Schiefner-Rohs 2018; Bresges 2018; Drossel und Eickelmann 2019) deren Ergebnisse und Folgerungen durch die hier vorliegende Evaluationsstudie gestützt werden.

\section{Zur Untersuchung}

Das Projekt «tabletBS» ist fokussiert auf Berufliche Gymnasien mit unterschiedlichen Fachprofilen in Baden-Württemberg und ist als fünfjähriger Schulversuch angelegt, der von den Autorinnen wissenschaftlich begleitet wird. Das Ziel der wissenschaftlichen Begleitung besteht darin, für den formalen Bildungskontext der Oberstufe an Beruflichen Gymnasien in Baden-Württemberg Antworten auf die folgende, vorwiegend deskriptiv angelegte, leitende Forschungsfrage zu geben: «Auf welche Art und Weise können Tablets zur Gestaltung von Lernprozessen - auch im Sinne der individuellen Förderung - in einzelnen Fächern eingesetzt werden?» 
Ausgangspunkt ist, dass sich Schulen auf eine Förderung beim Land bewerben konnten. Die teilnehmenden Schulen werden finanziell bei der Anschaffung von Tablets für einzelne Klassen unterstützt und erhalten Unterstützungsangebote bei der Implementierung. Dabei haben die Schulen selbst die Möglichkeit zu entscheiden, welches von drei Betriebssystemen und welchen Hersteller sie bevorzugen. Noch wichtiger als die Geräteauswahl erscheint aber die freie Ausgestaltung des Unterrichts mit den Geräten. Die Tablets sollen im Sinne einer 1:1-Ausstattung eingesetzt werden. Vorgaben zur Integration der Geräte konkret im Unterricht werden nicht gemacht, die Schulen respektive die Lehrerinnen und Lehrer sind von Seiten des Ministeriums für Kultus, Jugend und Sport Baden-Württemberg hier autonom agierend. Durch diese Freiheit ergeben sich pädagogische und didaktische Chancen für die teilnehmenden Schulen und Klassen mit ihrem jeweiligen Fachunterricht.

Evaluiert werden insgesamt rund 144 Klassen aus 40 Schulen in drei Tranchen à ca. 12 Schulen pro Jahr, die wiederum über die dreijährige Zeit der Oberstufe begleitet werden. Die nachfolgend in Abschnitt 4 dargestellten aggregierten Ergebnisse der sogenannten Entwicklungsfragebögen (E-Bogen), die jeweils zum Schuljahresende erhoben wurden, basieren auf den Angaben der folgenden jährlichen Teilnehmendenzahlen (N) an den EFragebögen:

\begin{tabular}{|l|l|l|l|l|}
\hline TN/Schuljahr & $2015 / 16(E 1)$ & $2016 / 17(E 2)$ & $2017 / 18(E 3)$ & 2018/19 (E4) \\
\hline $\begin{array}{l}\text { Lehrerinnen und } \\
\text { Lehrer }\end{array}$ & 108 & 187 & 240 & 117 \\
\hline $\begin{array}{l}\text { Schülerinnen } \\
\text { und Schüler }\end{array}$ & 484 & 884 & 1179 & 786 \\
\hline
\end{tabular}

Tab. 1.: Angaben der $\mathrm{N}$ für die Entwicklungsbögen (E-Bogen).

Zudem wurden ergänzend im Frühjahr 20163 Gruppendiskussion, im Jahr 2016/2017 12 leitfadengestützte Einzelinterviews mit Lehrerinnen und Lehrern, 2018 von Sommer bis Winter 14 leitfadengestützte Telefoninterviews mit Mitgliedern von Schulleitungen durchgeführt und 2019 von Frühjahr bis Sommer 6 eintägige Schulbesuche mit insgesamt 6 exemplarische Unterrichtsbeobachtungen und Gruppendiskussionen unternommen. 
Das Evaluationsdesign basiert vor allem auf quantitativen Erhebungsmethoden in Form von Online-Fragebögen zur Erfassung aller beteiligten Perspektiven, die nach Bedarf mit qualitativen Erhebungsmethoden für ausgewählte Perspektiven und für vertiefende Fragestellungen ergänzt werden.

Die Forschungsfrage wird über die Laufzeit in einer standardisierten Längsschnittuntersuchung mittels Online-Fragebögen untersucht. Zum Start einer Kohorte wird zum Schuljahresbeginn in den an «tabletBS» beteiligten Klassen jeweils einmalig eine IST-Erhebung aus Perspektive der Lehrerinnen und Lehrer und Schülerinnen und Schüler durchgeführt. Zum jeweiligen Schuljahresende wird im Juli ein Entwicklungsfragebogen (E-Bogen) über alle zum jeweiligen Zeitpunkt beteiligten Klassen für die Lehrerinnen und Lehrer und Schülerinnen und Schüler ausgegeben, der über die Zeit weitestgehend unverändert blieb, um vergleichende Aussagen treffen zu können. Für die früher abschliessenden Abiturjahrgänge wird der E-Bogen vorgezogen und als eigene Befragung kurz vor dem Abschluss ausgegeben (vgl. für einen Überblick Abb. 2).

\section{Forschungsdesign}

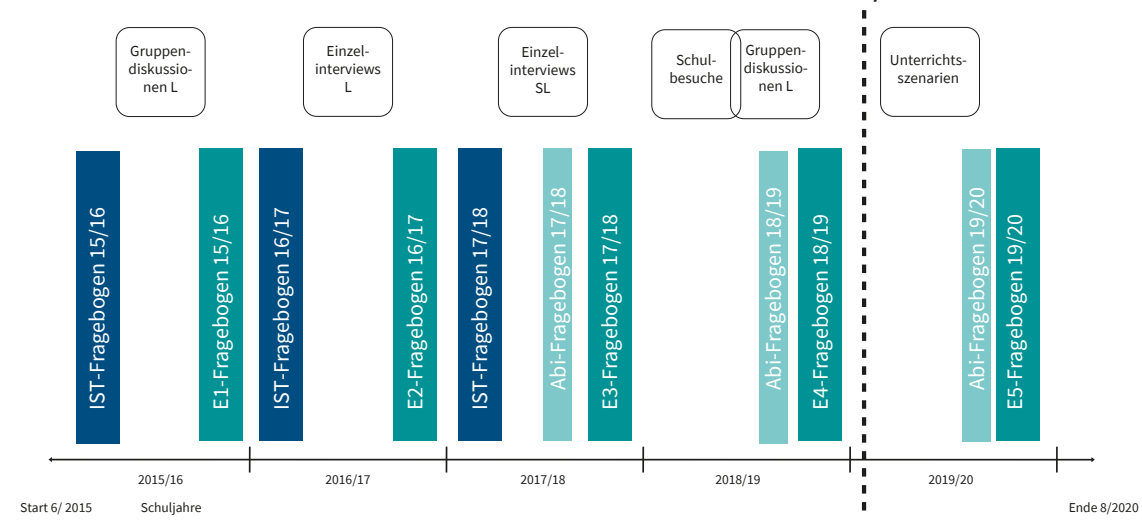

Zwischenstand 2018/19

Abb. 1.: Forschungsdesign über den Projektverlauf mit nicht- und standardisierten Erhebungsformen. 


\section{Ausgewählte Ergebnisse}

Nachfolgend werden ausgewählte Ergebnisse von «tabletBS» mit Fokus auf das Schuljahr 2018/19 sowie deren Entwicklung über die bisherige Projektlaufzeit dargestellt, die einen Überblick über die Projektentwicklung entlang der leitenden Fragestellung gewähren. Sie basieren im Wesentlichen auf den Ergebnissen der E-Bögen und geben Aufschluss hinsichtlich grundsätzlicher Entwicklungstendenzen über die Projektlaufzeit über alle Schulen hinweg.

Es wird dabei auf die folgenden Aspekte eingegangen: Nutzung und Akzeptanz, Unterrichtsgestaltung, Individualisierung, Kooperation, Motivation, Lernerfolg, Pädagogische Konzepte, Unterstützung und Projekteinbindung.

An dieser Stelle sei ausdrücklich darauf hingewiesen, dass die Evaluation von «tabletBS» nicht als Kompetenz- und Lernentwicklungserhebung angelegt ist, sowie auf Grund der gebotenen Anonymisierung personen-, klassen- oder schulbezogene Aussagen oder gar Wirkungen nicht explizit erhoben wurden. Zudem ist zu berücksichtigen, dass sich die Gruppe der teilnehmenden Lehrerinnen und Lehrer sowie Schülerinnen und Schüler (N) an den anonymisierten Befragungen je Fragebogen-Durchlauf in Anzahl und Projekterfahrung unterschiedlich zusammensetzt, so dass keine personen-, klassen- oder schulgebundenen Aussagen über Verläufe vorgenommen werden können.

\subsection{Zur Nutzung und Akzeptanz von Tablets im Unterricht}

Im Kontext der Mediennutzung zeigt sich, dass Lehrerinnen und Lehrer ihre digitalen Medien überwiegend privat und für schulische Belange gleichermassen nutzen (knapp 70 \%) wogegen umgekehrt die Schülerinnen und Schüler ihre digitalen Medien zunehmend überwiegend privat nutzen (über $60 \%$ ) und nur etwa ein Drittel (rund 34 \%) für schulische und private Belange.

Hieraus lässt sich schliessen, dass sich die Mediennutzung von Lehrenden und Lernenden im Fokus zwischen privaten und schulischen Belangen unterscheidet. Eine mögliche Folgerung ist daher, dass heutige Lehrerinnen und Lehrer zunehmend selbstverständlich und professionell digitale 
Kommunikations- und Informationsmedien nutzen - und mitunter auf Grund schulinterner Vorgaben nutzen müssen -, um Schulalltag und Unterricht vorzubereiten und zu organisieren. Das Wissen um diese unterschiedlichen Perspektiven von Schülerinnen und Schülern und Lehrerinnen und Lehrern bei der Mediennutzung ist hilfreich für die Gestaltung von lebensweltorientierten Lernumgebungen im Unterricht mit Tablets, um einerseits an Nutzungsgewohnheiten der Lernenden anschliessen zu können (z.B. beim Lernen und Gestalten mit Medien) und andererseits bewusst neue Erfahrungsmöglichkeiten zu schaffen und zu erkunden (z.B. beim Lernen über Medien).

Fragt man die Lehrerinnen und Lehrer, die in den Projektklassen unterrichten, ob das Tablet bereits fest in ihren Unterricht integriert ist, so stimmen 2018/2019 in der letzten Befragung gut die Hälfte dieser Aussage zu, ein Viertel gibt an, es eher selten zu nutzen und $7 \%$, dass Tablets kaum oder gar nicht genutzt werden (s. Tab. 2).

\begin{tabular}{|l|l|l|l|l|l|}
\hline & IST1 & E1 & E2 & E3 & E4 \\
\hline Ja, es wird sehr häufig genutzt. & $41 \%$ & $52 \%$ & $46 \%$ & $47 \%$ & $54 \%$ \\
\hline Es wird eher selten genutzt. & $32 \%$ & $34 \%$ & $33 \%$ & $33 \%$ & $25 \%$ \\
\hline $\begin{array}{l}\text { Nein, es wird kaum oder gar } \\
\text { nicht genutzt. }\end{array}$ & $9 \%$ & $5 \%$ & $5 \%$ & $5 \%$ & $7 \%$ \\
\hline
\end{tabular}

Tab. 2.: (Lehrerinnen/Lehrer): Haben Sie das Tablet bereits fest in Ihren Unterricht integriert? (N= IST1:136; E1: 108; E2: 187; E3: 240; E4: 117; fehlende Wert zu 100: k. A.).

Eine entsprechende Tendenz lässt sich auch hinsichtlich der Frage nach der Freude daran, das Tablet methodisch im Unterricht zu integrieren, ablesen (s. Tab. 3). Hier zeigen ca. 40 \% der Lehrerinnen und Lehrer klar auf, dass sie Freude daran haben; ein Drittel (34 \%), dass sie sowohl gerne mit als auch ohne Tablet unterrichten und lediglich $10 \%$, dass ihnen ihre bisherige Unterrichtsweise ohne Tablet mehr Freude bereite. 


\begin{tabular}{|l|l|l|l|l|l|}
\hline & IST1 & E1 & E2 & E3 & E4 \\
\hline $\begin{array}{l}\text { Ja, es macht mir Freude die Tab- } \\
\text { lets methodisch in meinen Unter- } \\
\text { richt zu integrieren. }\end{array}$ & $59 \%$ & $60 \%$ & $67 \%$ & $39 \%$ & $39 \%$ \\
\hline $\begin{array}{l}\text { Nein, meine bisherige Unterrichts- } \\
\text { weise bereitet mir mehr Freude. }\end{array}$ & $4 \%$ & $15 \%$ & $7 \%$ & $6 \%$ & $8 \%$ \\
\hline $\begin{array}{l}\text { Ich unterrichte sowohl mit dem } \\
\text { Tablet als auch ohne gleich gerne. }\end{array}$ & $6 \%$ & - & - & $40 \%$ & $34 \%$ \\
\hline Kann ich (noch) nicht beurteilen. & $15 \%$ & - & - & $3 \%$ & $7 \%$ \\
\hline
\end{tabular}

Tab. 3.: (Lehrerinnen/Lehrer): Haben Sie Freude daran, das Tablet als neues Medium in Ihrem Unterricht methodisch zu integrieren? (N= IST1:136; E1: 108; E2: 187; E3: 240; E4: 117; fehlende Wert zu 100: k. A.).

Insgesamt lässt sich im Verlauf bis heute feststellen, dass über die Zeit mit steigender Tendenz zwei Drittel der Lehrenden der Integration des Tablets in den Unterricht grundsätzlich aufgeschlossen gegenüber stehen; etwa ein Drittel steht den Entwicklungen neutral gegenüber und gute $10 \%$ teilen die positive Perspektive nicht.

\subsection{Zur Rolle von Tablets für die Veränderung der Unter- richtsgestaltung}

Im Zuge von «tabletBS» zeigt sich bisher, trotz einzelner herausragender didaktisch innovativer Beispiele für mobiles Lernen, dass die Tablets überwiegend als Ergänzung und Erweiterung des bisherigen Fachunterrichts eingesetzt werden. Das heisst, so konnte es den optionalen Freitexterläuterungen der standardisierten Fragen entnommen werden, dass die Tabletintegration zumeist im bestehenden didaktischen Konzept des Unterrichts unter Ausnutzung neuer Funktionen und Angebote sowie methodischer Zugänge (Feedback, Quiz, Kollaboration) stattfindet. Es zeichnet sich in den unterschiedlichen Erhebungen und vor allem Interviews ab, dass der Tableteinsatz als Möglichkeit gesehen wird, neue Funktionen und Angebote sowie neue methodische Zugänge zu nutzen. Das Tablet wird in diesem Zusammenhang zunehmend für unverzichtbar gehalten (von anfänglich $26 \%$ auf derzeit $36 \%$.) 
In den optionalen Freitexten wurden als Gründe für eine Verzichtbarkeit bspw. angegeben, dass ein guter Unterricht von Lehrendenkompetenzen und nicht von Hilfsmitteln wie Tablets abhänge oder dass das Tablet Aufgaben, wie Aufsatzschreiben, nicht abnehmen könne. Zudem wurde auf andere etablierte Hilfsmittel verwiesen. Ebenso wurden Gründe angeführt für die mittlerweile wahrgenommene Unverzichtbarkeit von Tablets im Unterricht. So werden sie bspw. als gute Möglichkeit gesehen, den Unterricht abwechslungsreich zu gestalten, ein digitales Klassenbuch leichter handhabbar zu führen sowie grundsätzlich mit Tablets zeitgemässen Unterricht durchzuführen. Ein solcher zeitgemässer Unterricht wird häufig auch mit einem «anderen» Unterricht in Verbindung gebracht, wie er von mehr als der Hälfte der Lehrerinnen und Lehrer im Zuge von «tabletBS» gestaltet wird.

Auf die Frage nach einer anderen Gestaltung des Unterrichts mit dem Tablet, verglichen zu der Unterrichtsgestaltung in einer Klasse ohne Tablets, beschreiben die Lehrenden in ergänzenden optionalen Freitextkommentaren, was für sie ein anderes Lernen und Lehren ausmacht. Dazu gehören beispielsweise eine stärkere Lernendenzentrierung, mehr Projektarbeit, längere Lernenden-Arbeitsphasen, andere vielfältigere Feedbackmöglichkeiten, Flipped Classroom, Gamification, selbstständige Internetrecherche, weniger Frontalunterricht. Für die Unterrichtsgestaltung lassen sich folgende Beispiele benennen: Lernvideos erstellen, Internetrecherche während des Unterrichts passgenau durchführen, gemeinsame Erstellung von E-Books, kollaboratives Arbeiten mit dem Tool «Padlet» für Gruppenpuzzle oder Pro/Contra-Diskussionen.

\subsection{Zur Rolle von Tablets für die individuelle Förderung}

Die unterschiedlichen Befragungen über die bisherige Laufzeit von «tabletBS» zeigen, dass eine Sensibilisierung und aktive Integration des Konzepts einer individuellen Förderung (potenziell) dazu beiträgt, dass eine räumliche Reorganisation des Unterrichts stattfindet, die wiederum zu einer Verschiebung vom Frontalfokus auf einen Kooperationsfokus beiträgt, der eine mitunter individuellere Betreuung ermöglicht. Eine Veränderung der Lehrendenrolle erfolgt zumeist, indem mehr 
Kooperationsmöglichkeiten entstehen, u.a. das gemeinsame Finden von Lösungen von technischen Problemen mit den Geräten zwischen den Lernenden oder auch zwischen Lehrenden und Lernenden, was wiederum zur positiven Beziehungsarbeit beitragen kann. Die Diagnose von Stärken und Schwächen durch Tool-Unterstützung (z.B. Teams) wird einfacher möglich (u.a. Option des direkten individuellen Feedbacks durch Apps), wodurch sich insgesamt ein niveaudifferenziertes Arbeiten (z.B. durch auf die Schülerinnen und Schüler angepasste Aufgabenniveaus) leichter ermöglichen lässt. Ebenso tragen vielfältige Feedbackoptionen (u.a. mehr Zeit für die einzelnen Lernenden, mit Audio-Apps eingesprochenes, persönlicheres Feedback) zur Beziehungsstärkung bei. Gleichzeitig zeigt sich aber, dass das Verständnis vom Konzept individueller Förderung divers ist und von reiner Binnendifferenzierung bis zu individueller Einzelbetreuung reicht.

Es wird deutlich, dass seit Projektbeginn mittlerweile niemand mehr angibt eher selten oder nie die Möglichkeiten zu haben, die Lernenden im Unterricht individuell zu fördern (von knapp einem Viertel gesunken auf ca. $2 \%$ der befragten Lehrenden).

Die Hälfte der Lehrenden ist der Auffassung, dass das Tablet ein nützliches Hilfsmittel ist, um vermehrt zur individuellen Förderung beizutragen.

Parallel dazu wurde die Perspektive der Schülerinnen und Schüler im weitesten Sinne auf das Thema Individualisierung erfragt, inwiefern seit Beginn des Tabletprojektes mehr auf ihre individuellen Bedürfnisse eingegangen wird (s. Tab. 9). Hier geben durchweg etwa $10 \%$ der Lernenden an, dass sie keinen verbesserten Eindruck haben, aber knapp zwei Drittel, dass manchmal oder immer bzw. meistens eine höhere Individualisierung stattfindet. Es zeigt sich, dass Tablets von Lehrenden wie Lernenden als unterstützend für Aktivitäten zur Individualisierung wahrgenommen werden und sich so Optionen erweitern (u.a. Passung von Aufgabenstellungen, Diagnose, Feedback). Eine weitere Verständigung über ein einheitliches Verständnis über das Konzept von Individualisierung kann helfen, noch gezielter entsprechende Unterrichtsszenarien zu entwickeln. 


\subsection{Zur Rolle von Tablets für die Kooperation (Lehrerinnen/ Lehrer)}

In der Gesamtdatenlage und vor allem in den mündlich erhobenen Daten zeigt sich, dass solche Lehrerinnen und Lehrer, die man als sehr engagiert im Projektzusammenhang bezeichnen kann, durchweg kooperativ eingestellt sind und in dieser Weise auch arbeiten. Kooperation unter den Lehrerinnen und Lehrern zeigt sich je Schule beispielsweise daran, dass die Organisation und das Teilen von Unterrichtsmaterial über unterschiedliche technische Wege und Plattformen erfolgt. Eine Kooperation zwischen den Lehrkräften erfolgt zudem durch regen Austausch innerhalb der Fachschaften. Besonders relevant ist die bedarfsorientierte Organisation von Massnahmen zur internen Unterstützung untereinander (z.B. Workshops), wobei sich als etabliertes Konzept der Kooperation der Austausch auf kurzem Weg oder «auf dem Flur» durchgesetzt hat. Zumeist finden keine festgelegten und verpflichtenden regelmässigen schulinternen Fortbildungsmassnahmen statt. In den Interviews zeigt sich auch, dass die Unterrichtsentwicklung vor Ort besonders vom Engagement der Lehrenden und ihrer Bereitschaft (zusätzliche) Zeit in die Kooperation zu investieren, abhängt.

Die Kooperation der Lehrenden erfolgt vornehmlich innerhalb der Einzelschule und bislang kaum schulübergreifend mit anderen Tablet-Schulen.

\subsection{Zur Rolle von Tablets für die Motivation}

Auf Basis der vorliegenden Daten können in der Tendenz vorsichtige Aussagen zur Rolle von Tablets für die Motivation im Unterricht aus Perspektive der Lehrerinnen und Lehrer sowie der Schülerinnen und Schüler getroffen werden. Lehrende und Lernende haben eine ähnliche Sichtweise auf die Förderung durch Tablets im Hinblick auf die Motivation: etwa ein Drittel schreibt ihnen eine Motivationsförderung zu und etwa die Hälfte kann keine Veränderung erkennen bzw. macht die Motivation nicht vom Tableteinsatz, sondern von anderen Faktoren abhängig.

Lehrende wie Lernende sehen Ablenkung im Unterricht als Thema an. Dabei werden von den Lernenden eher andere Gründe, die zur Ablenkung führen, angeführt als allein der Tableteinsatz, während Lehrende das Tablet stärker als Verursacher sehen. 


\subsection{Zur Rolle von Tablets für den Lernerfolg}

Im Zuge von «tabletBS» können zu Fragen des individuellen Lernerfolgs durch den Tableteinsatz im Unterricht keine personengebundenen Angaben gemacht werden oder lehrenden- oder lernendengebundene Entwicklungslinien aufgezeigt werden. In den Selbsteinschätzungen von Lehrerinnen und Lehrern sowie Schülerinnen und Schülern, die sich im jeweiligen Jahr an der Umfrage beteiligt haben, zeigt sich über die Projektlaufzeit, dass etwa zwei Drittel der Lehrenden und Lernenden dem Tablet grundsätzlich eher eine förderliche Rolle hinsichtlich fachlicher und überfachlicher Kompetenzen zuschreiben. Doch zugleich zeigt sich auch, dass Lehrende wie Lernende andere (hier nicht weiter erfragte) Faktoren für den Lernerfolg höher bewerten als den Tableteinsatz mit Tools im Unterricht. Unbenommen dessen, wird das Tablet von den Lernenden sehr deutlich als hilfreich für das Lösen von Aufgaben angesehen und trägt so zum erfolgreichen Lernprozess bei (s. Tab. 3).

\begin{tabular}{|l|l|l|l|l|l|}
\hline & IST1 & E1 & E2 & E3 & E4 \\
\hline Ja, es hilft mir sehr oft. & - & $21 \%$ & $23 \%$ & $26 \%$ & $29 \%$ \\
\hline Ja, es hilft mir manchmal. & - & $54 \%$ & $47 \%$ & $48 \%$ & $44 \%$ \\
\hline Es hilft mir eher selten. & - & $11 \%$ & $10 \%$ & $10 \%$ & $10 \%$ \\
\hline $\begin{array}{l}\text { Nein, ich löse Aufgaben- und Pro- } \\
\text { blemstellungen ohne das Tablet. }\end{array}$ & - & $3 \%$ & $4 \%$ & $4 \%$ & $5 \%$ \\
\hline
\end{tabular}

Tab. 4.: (Schülerinnen/Schüler): Hilft Ihnen das Tablet im Unterricht dabei, Aufgaben oder Problemstellungen zu lösen? (N= IST1:575; E1: 484; E2: 884; E3: 1179; E4: 786; fehlende Wert zu 100: k.A.).

\subsection{Zur Rolle von Tablets für (medien-)pädagogische Konzep- te an den Schulen}

Aus den Interviews und Unterrichtsbesuchen mit anschliessenden Diskussionen konnte entnommen werden, dass die Erarbeitungsphase eines $\mathrm{Me}$ dienkonzepts bzw. die Einbindung in das pädagogische Konzept der Schule vielerorts noch im Gange ist. Dabei zeigt sich, dass medienpädagogische Aspekte nicht immer zugleich Teil der weiterreichenden pädagogischen (Schul-)Konzepte sind. Dies erklärt sich damit, dass im bisherigen Projektverlauf der Fokus vielerorts auf die Schaffung grundlegender Strukturen 
für das Projekt und deren Funktionieren im Schul- und Unterrichtsalltag gelegt wurde. So stand vor allem die Herstellung einer funktionierenden Infrastruktur im Zentrum, wozu auch die Organisation von (regelmässig verfügbarer) technischer Unterstützung innerhalb der Schulen oder durch Kooperationen zählt. Daneben brauchte es eine Einigung und Implementierung auf ein einheitliches Ablagesystem für Materialien (innerhalb der einzelnen Schule), so dass mit Online-Materialien über das W-Lan in der Schule gearbeitet werden kann. Als grösste Herausforderung für die Konzeptentwicklung und den Unterrichtsalltag werden nach wie vor die Problematik mit dem Datenschutz und Fragen von Nutzungsrechten bei Unterrichtsmaterialien benannt, was zur Zurückhaltung beim Austausch über das Schulkollegium hinaus führt. Entsprechend stellt das IT- und Datenmanagement einen Hemmnisfaktor dar, weshalb vielfach der deutliche Wunsch nach einer rechtlich sicheren Schulcloud bestärkt wurde.

\subsection{Projekteinbindung, -organisation und Vernetzung}

Ob Unterstützungsbedarfe im Unterricht und an den Schulen, Unterstützungssysteme an den Schulen und durch die Kolleginnen und Kollegen sowie darüber hinaus, existieren, hängt mit der Form der Projekteinbindung, -organisation und Vernetzung der beteiligten Akteurinnen und Akteure zusammen. Zentraler Aspekt für das Gelingen der Implementierung und nachhaltigen Verankerung von didaktischen und technischen Lehrinnovationsprojekten wie «tabletBS» eines ist, sind die Schulleitungen. Etwa zwei Drittel der Lehrerinnen und Lehrer geben an, dass sie sich gut unterstützt fühlen bei der Einführung der Tablets in den Unterricht (s. Tab. 5). Dabei zeigt sich auch, dass die Unterstützung während der Einführungsphase des Projekts durch Schulleitung und Projektverantwortliche von Lehrenden höher eingeschätzt wird als im Projektverlauf. 


\begin{tabular}{|l|l|l|l|l|l|}
\hline & IST1 & E1 & E2 & E3 & E4 \\
\hline Ja & $61 \%$ & $62 \%$ & $65 \%$ & $66 \%$ & $60 \%$ \\
\hline Nein & $2 \%$ & $9 \%$ & $6 \%$ & $6 \%$ & $15 \%$ \\
\hline
\end{tabular}

Tab. 5.: (Lehrerinnen/Lehrer): Fühlen Sie sich bei der Einführung der Tablets in den Unterricht durch die Schulleitung bzw. die Projektverantwortlichen gut unterstützt? (N= IST1:136; E1: 108; E2: 187; E3: 240; E4: 117; fehlende Wert zu 100: k. A.).

Die Lernenden wiederum fühlen sich bei der Nutzung der Tablets durchweg eher gut unterstützt. Insgesamt zeigt sich für Lehrende wie Lernende, dass ein Bedarf an stetigen und systematischen Unterstützungsangeboten besteht. Dies betrifft sowohl den Bereich der Funktionalität der Infrastruktur, wie auch den Bereich der medienbezogenen Kompetenzen der Lehrenden und Lernenden sowie den Bereich der fachlichen und didaktischen Unterrichtsentwicklung mit Tablets. In den Gesprächen zeigt sich eine hohe Aufgeschlossenheit gegenüber einer stetigen Konzept- und Netzwerkarbeit, da diese als sinnvoll und zielführend eingeschätzt wird und erwünscht ist.

Die hier aufgezeigten Entwicklungen bestätigen sich auch durch Faktoren hinsichtlich der Unterstützungsprozesse, die von den interviewten Schulleitungsmitgliedern benannt wurden. Sie gehen ebenso auf Aspekte der Projekteinbindung, -organisation und Vernetzung der beteiligten Akteurinnen und Akteure ein. So lassen sich zu den bisher angeführten Punkten noch folgende Aspekte aufzeigen, die als Gelingensbedingungen betrachtet werden. Dazu gehören die Beachtung von Vorlaufzeiten, die (technische) Einführung aller Beteiligten in die Arbeit mit Tablets sowie fachspezifische Fortbildungen von Lehrenden, ausserdem das Vorhandensein von Infrastruktur wie auch digitalem oder Online-Unterrichtsmaterial. Aus Perspektive der Schulleitungen kommt aber auch die Auseinandersetzung mit Arbeitsbelastungen und der Kostenfaktor hinzu, also der umfassende Blick auf die Ressourcen. Zudem wird gesehen, dass eine positive Haltung der Lehrenden zum Einsatz von Tablets förderlich ist, dass positive Beispiele helfen, Skepsis zu mindern und Prozesse auch Geduld brauchten, bis sie in alltägliche Routinen überführt seien. Wichtig sei es dabei auch, die Schülerschaft abzuholen und das Kollegium in Entscheidungsprozesse miteinzubeziehen. 


\section{Zusammenfassung und Gesamteinschätzung}

Innerhalb der Einzelkapitel wurden bereits themenspezifische Zusammenfassungen mit ersten Folgerungen sowie Beispiele ausgeführt. An dieser Stelle erfolgt nun eine Zusammenfassung und Bewertung der bis heute im Projekt «tabletBS» vorliegenden Ergebnisse mit Blick auf die im schulischen Kontext vielfach erörterte Frage nach dem Mehrwert bei der Gestaltung von Lernprozessen mit Tablets im Allgemeinen und im Sinne der individuellen Förderung aus pädagogischer, didaktischer, medienbezogener sowie fachlicher Perspektive. Nachfolgend werde dieser Mehrwert erörtert (5.1) sowie Aufgaben für die weitere Arbeit benannt (5.2).

\subsection{Zum Mehrwert bei der Gestaltung von Lernprozessen mit Tablets im Allgemeinen und im Sinne der individuellen Förderung}

Aus pädagogischer und allgemeindidaktischer Perspektive zeigen sich im Projektverlauf folgende pädagogische wie didaktische Vorteile der Tabletintegration: Es lässt sich nach Selbsteinschätzung der Lehrenden und Lernenden grundsätzlich eine Förderung überfachlicher Kompetenzen bzw. Schlüsselkompetenzen (u.a. Problemlösefähigkeit, Teamarbeit, Projektmanagement, Kommunikation) feststellen.

In der Breite zeigt sich, dass das Tablet vorwiegend als Werkzeug und Substitution gegenüber bisher analog verwendeter Hilfsmittel eingesetzt wird. Das bestehende didaktische Konzept wird zumeist mit Tablets erweitert, doch nicht grundlegend neu gedacht. Erst wenige Lehrende verändern die didaktischen Konzepte mit Blick auf die nunmehr erweiterten Möglichkeiten deutlich und praktizieren ein mobiles Lernen. Hier zeigt sich dann in besonderer Weise, dass sich «neue» Lernmethoden zur Förderung einer Lernendenorientierung integrieren lassen und dass mit individuell zur Verfügung stehenden, vernetzten Tablets eine Vereinfachung von Kollaboration und Kooperation im Unterricht möglich ist. Insgesamt ermöglichen Tablets eine räumliche wie soziale Öffnung von Unterricht und tragen so förderlich zur Beziehungsgestaltung zwischen Lehrenden wie Lernenden und zwischen den Lernenden untereinander bei. Es lässt sich eine stetige Akzeptanzsteigerung in der Breite bei einem Grossteil der 
Lehrenden und Lernenden gegenüber Tablets im Unterricht verzeichnen. Hier schliesst an, dass eine zunehmende Ausschöpfung von didaktischen und pädagogischen Potenzialen mobiler Geräte im Unterricht erfolgt. Damit geht einher, dass Tablets tendenziell hohes Potenzial für eine zunehmende, individualisierte Unterrichtsgestaltung beigemessen werden kann. Auch zeigt sich, dass Tablets bisherige Aktivitäten zur Individualisierung unterstützen und die Optionen erweitern. In der didaktischen Gestaltung von Lernszenarien mit Tablets liegt ein besonderes pädagogisches Potenzial für eine Individualisierung, insbesondere, wenn eine Verständigung über das gemeinsame Konzept von Individualisierung zielführend unterstützt wird. Bisherige Ergebnisse zeigen, dass gerade die Förderung der Beziehungsarbeit im Unterricht zwischen Lehrenden und Lernenden durch eine veränderte Raumgestaltung vielfältige Möglichkeiten bietet. So kann eine Frontalsituation hin zu einer stärkeren Zugewandtheit aufgelöst werden, damit der kooperative und kollaborative Fokus, den Tablets unterstützen, gestärkt wird.

Aus fachlicher bzw. fachdidaktischer Perspektive zeigen die Projektergebnisse, dass nach Selbsteinschätzung der Lehrenden und Lernenden fachliche Kompetenzen und besonders das Bearbeiten von Aufgaben positiv unterstützt werden. Im Zuge von Aufgabenbearbeitungen zeigt sich ein grosser Vorteil hinsichtlich der Einbindung von Aktualitätsbezügen und das Ermöglichen von kritischen Perspektiven auf Inhalte auf Grund der vielfältig vorliegenden und recherchierbaren Informationen. Zugleich sind Lehrende wie auch Lernende so einer stetigen fachlichen Überprüfbarkeit ausgesetzt. Ein veränderter Umgang mit fachlichen Informationen und deren Qualität, wie beispielsweise in der KMK-Strategie für eine digitale Welt gefordert (s. KMK 2017, 12) kann durch den Einsatz von Tablets mitbearbeitet werden, so dass hier im Querschnitt fachliche und medienbezogene Kompetenzen gleichermassen gefördert werden. Zugleich findet im Fachunterricht auch eine Förderung von fachlichen wie überfachlichen Individualisierungstendenzen statt.

Aus medien- und technologiebezogener Perspektive lässt sich festhalten, dass der Tableteinsatz zu einer Steigerung der Sicherheit und Souveränität im alltäglichen Medienumgang mit den Tablets (u.a. Unterrichtsorganisation, Kommunikation) bei Lehrenden und Lernenden führt. So hat sich 
gerade in technischer Hinsicht eine stetige positive Haltung bei einem Grossteil der Lehrenden in der Breite und über die Zeit entwickelt, nachdem es anfängliche «Startschwierigkeiten» gab, die nun überwunden sind. Dies führt auch dazu, dass kleinere technische Herausforderungen nicht mehr als unüberwindbare Probleme betrachtet werden, sondern tendenziell eine positive Entwicklung einer offenen und pragmatischen Haltung im Umgang mit Tablets im Unterrichtsalltag bei Lehrenden und Lernenden zu verzeichnen ist und ein gemeinsames Problemlösen sowie eine stärkere technologiebezogene Routine beim Einsatz von Tablets festzustellen ist. So ist ein fortdauernder Prozess einer Verstetigung hinsichtlich der alltäglichen Integration der Tablets erkennbar.

Die hier dargestellten pädagogischen, didaktischen, medienbezogenen sowie fachlichen Perspektiven auf die Integration von Tablets im Unterricht bzw. mobilem Lernen decken sich mit dem Stand der medien- wie fachdidaktischen Fachdiskussion.

\subsection{Folgerungen}

Durch die Entwicklungsdaten 2018/2019 zeigt sich eine leicht rückläufige Tendenz nach vier Jahren von positiven Trends. Eine mögliche Erklärung kann hierbei sein, dass die erste Kohorte der vermeintlich vermehrten Enthusiasten nach nunmehr drei Jahren nicht mehr systematisch an der Erhebung teilnimmt, da die Förderung an den ersten Schulen ausgelaufen ist. Es lässt sich hier die Annahme formulieren, dass dem Modell von Innovationsdiffusionen nach Rogers für den Schulkontext folgend (s. Schaumburg, Prasse und Blömeke 2009), die sogenannten Early Adopters und Teile der Early Majority sich mit den unterschiedlich startenden Zeitpunkten der drei Tranchen leicht verschoben haben. Sprich, waren vermutlich bei der ersten Kohorte, die in den Jahren 2015-2018 untersucht wurde, eher überwiegend motivierte Enthusiastinnen und Enthusiasten dabei, wäre es möglich, dass ab der dritten Tranche mit Start 2017 vermehrt solche Kolleginnen und Kollegen dabei sind und zum derzeitigen Zeitpunkt die Datenlage im Schwerpunkt prägen, die sich von der Idee haben über die Zeit überzeugen lassen, doch nach wie vor mit einem pragmatischen Ansatz und Blick teilnehmen. Diese Grundhaltung könnte eine Erklärung für 
die leichte Veränderung bei einigen Tendenzen innerhalb der untersuchten Bereiche sein, da jede Person eine persönliche Zeitspanne benötigt, um eine Akzeptanzentwicklung zu durchlaufen.

Inwiefern sich diese Tendenz bestätigt, werden die Ergebnisse der Abschlusserhebung 2020 zeigen, die nochmals an alle Teilnehmenden, die sich zu dem Zeitpunkt noch im Projekt befinden, ausgegeben wird. Unbenommen dessen, zeigen die Ergebnisse in besonderem Mass, dass gerade bei einem so lange laufenden Projekt es stetig die Aufgabe ist und sein sollte, über die gesamte Laufzeit die Nachjustierung von Zielen, Konzepten, Verbindlichkeiten und Zuständigkeiten, wo es sinnvoll erscheint, vorzunehmen und im Sinne einer nachhaltigen Verankerung zu begleiten. Trotzdem die Projektlaufzeit fast geendet ist, erscheint es gerade mit Blick auf die späteren Aktivitäten wichtig, (auch vorsorglich) das besondere Engagement der Lehrenden und Schulen weiterhin wertzuschätzen, um so auch zur weiteren Motivation beizutragen und zu verdeutlichen, den Weg weiter gehen zu wollen.

Die Ergebnisse zeigen auch auf, dass eine weitere Verständigung über die Rolle von Individualisierung und deren Formen in Unterricht und Schule wichtig bleibt, damit dieses zentrale Thema im Unterricht und bei der Entwicklung von Unterrichtsszenarien mit Tablets die angemessene Beachtung findet.

Eine vielfach artikulierte Sorge wird im Bereich der technischen Sicherheit (u.a. Cloudlösung) geäussert und es bestehen Hemmnisse bei (nutzungs-)rechtlichen Rahmenbedingungen (u.a. für schulübergreifenden Tausch von Unterrichtsmaterial). Für Letztere Lösungen zu finden, stellen Aufgaben auf Landesebene dar.

Bezogen auf die Schulen wird deutlich, dass eine massgebliche Bedingung für die gelingende (medien-) pädagogische Arbeit, idealerweise die nachhaltige (schulübergreifende) Verankerung von (medien-) pädagogischdidaktischen Konzepten mit Tablets in Schule und Unterricht ist.

Dabei zeigt sich als Aufgabe, dass weiterhin systematische Unterstützungsangebote im Bereich Unterrichtsentwicklung und (schulübergreifendem) Austausch mit Blick auf Verstetigung beizubehalten und auszubauen sowie, wo möglich, informelle Netzwerke zu fördern sind und je nach Bedarf personelle Ressourcen erweitert werden. 


\section{Ausblick und Fazit}

Mit Blick auf den vorliegenden Zwischenstand lässt sich ein Ausblick auf das weitere Vorgehen geben und bereits ein knappes Fazit ziehen.

\subsection{Ausblick}

Im letzten Jahr des Projekts «tabletBS» wird, um dem Längsschnittdatensatz gerecht zu werden, erneut eine standardisierte Erhebung der Entwicklungen über das Jahr erfolgen. Der letzte Fragebogen wird dabei nochmals explizit an alle Projektbeteiligten über die fünf Jahre auf Seiten der Lehrenden ausgegeben, damit alle Perspektiven abgebildet werden.

Die qualitative Zwischenerhebung legt zum Projektabschluss den Fokus auf die Vielfalt an entstandenen Unterrichtsszenarien in den Fächern. Dafür wird eine systematische Erhebung im Laufe des Schuljahres 2019/20 erfolgen. Die Ergebnisse werden strukturiert aufbereitet und können als Orientierung für entsprechende Fortbildungen oder Handreichungen weiterverarbeitet werden.

\subsection{Fazit}

Es lässt sich nach vier Jahren Projektlaufzeit festhalten, dass

- Tablets stetig, positiven Einfluss auf die schülerorientierte Entwicklung der Unterrichtsgestaltung haben.

- eine gute Beziehungsgestaltung zwischen Lehrenden und Lernenden fördern.

- zunehmende Akzeptanz und Verstetigung der Tabletintegration im Unterricht stattfindet.

- es engagierte Lehrende braucht, die das Projektvorhaben in den Schulen wesentlich mitentwickeln.

- Unterstützungsangebote zur Unterrichtsentwicklung, Konzept- und Netzwerkarbeit erwünscht und zielführend sind.

Das Projekt «tabletBS» zeichnet in besonderer Weise die lange Förderdauer bzw. Projektlaufzeit aus, womit es sich von zahlreichen anderen Modellversuchen und Pilotunternehmungen absetzt. Es kann vor diesem 
Hintergrund festgehalten werden, dass die vorliegenden Ergebnisse nach einer längeren Projektlaufzeit den bisherigen Forschungsstand zur Implementierung und zum Einsatz mobiler Endgeräte im Unterricht bestätigen und stützen.

Die Aufgabe liegt nun darin, die positiven Entwicklungen der letzten vier Jahre über die nächsten Jahre auf den unterschiedlichen Ebenen zu verstärken, zu unterstützen und nachhaltig zu verankern.

\section{Literatur}

Bastian, Jasmin, und Stefan Aufenanger, Hrsg. 2017. Tablets in Schule und Unterricht: Forschungsmethoden und -perspektiven zum Einsatz digitaler Medien. Wiesbaden: Springer VS.

Bresges, André. 2018. «Mobile Learning in der Schule». In Handbuch Mobile Learning, herausgegeben von Claudia de Witt und Christina Gloerfeld, 613-35. Wiesbaden: Springer VS.

Drossel, Kerstin, und Birgit Eickelmann. 2019. «Potenziale der Tabletnutzung im Unterricht zur individuellen Förderung - Analysen und Forschungsperspektiven». In Individuelle Förderung im Unterricht. Empirische Befunde und Hinweise für die Praxis, herausgegeben von Hannelore Knauder und Christa-Monika Reisinger, 143-56. Münster: Waxmann.

Eickelmann, Birgit. 2019. «ICILS 2018: Vergleichsstudie zu digitalen Kompetenzen bei Schülern». Universität Paderborn. https://www.uni-paderborn.de/ nachricht/91521/.

Eickelmann, Birgit, Wilfried Bos, Julia Gerick, Frank Goldhammer, Heike Schaumburg, Knut Schwippert, Martin Senkbeil, Jan Vahrenhold, und Waxmann Verlag, Hrsg. 2019. ICILS 2018 \#Deutschland Computer- und informationsbezogene Kompetenzen von Schülerinnen und Schülern im zweiten internationalen Vergleich und Kompetenzen im Bereich Computational Thinking. Münster: Waxmann.

Galley, Kathrin, und Kerstin Mayrberger. 2018. «Tablets im Schulalltag: Potenziale und Herausforderungen bei der Integration von mobilen Endgeräten an beruflichen Gymnasien». MedienPädagogik 31: 36-57. http://dx.doi.org/10.21240/ mpaed/31/2018.03.27.X.

Gerick, Julia, und Birgit Eickelmann. 2018. «Mobiles Lernen und BYOD an Gemeinschaftsschulen. Voraussetzungen und Potenziale aus Sicht der Schulentwicklung». schulmanagement 3: 8-12.

Heinz, Susanne. 2018. Mobile Learning und Fremdsprachenunterricht: theoretische Verortung, Forschungsüberblick und Studie zum Englischunterricht in TabletKlassen an Sekundarschulen in Bayern. 1. Auflage. Beiträge zur historischen und systematischen Schulbuch- und Bildungsmedienforschung. Bad Heilbrunn: Julius Klinkhardt. 
KMK - Kultusministerkonferenz. 2017. «Strategie der Kultusministerkonferenz „Bildung in der digitalen Welt" Beschluss der Kultusministerkonferenz vom 08.12.2016 in der Fassung vom 07.12.2017». www.kmk.org/fileadmin/Dateien/ pdf/PresseUndAktuelles/2018/Digitalstrategie_2017_mit_Weiterbildung.pdf.

Mayrberger, Kerstin. 2018. «Rahmenbedingungen für die Gestaltung von Lernumgebungen mit mobilen Endgeräten». In Handbuch Mobile Learning, herausgegeben von Claudia de Witt und Christina Gloerfeld, 63-82. Wiesbaden: Springer VS. https://doi.org/10.1007/978-3-658-19123-8_4.

Schaumburg, Heike. 2018. «Empirische Befunde zur Wirksamkeit unterschiedlicher Konzepte des digital unterstützten Lernens». In Digitalisierung in der schulischen Bildung: Chancen und Herausforderungen, herausgegeben von Nele McElvany, Franziska Schwabe, Wilfried Bos, und Heinz Günter Holtappels, 27-41. IFS-Bildungsdialoge, Band 2. Münster; New York: Waxmann.

Schaumburg, Heike, Doreen Prasse, und Sigrid Blömeke. 2009. «Implementation von Innovationen in der Schule». In Handbuch Schule: Theorie - Organisation Entwicklung, herausgegeben von Sigrid Blömeke, Thorsten Bohl, Ludwig Haag, Gregor Lang-Wojtasik, und Werner Sacher, 560-601. Bad Heilbrunn: Julius Klinkhardt.

Scheiter, Katharina, und Andreas Lachner. 2019. «DigitalPakt - was nun? Eine Positionierung aus Sicht der Lehr-Lernforschung» Unterrichtswissenschaft 47(4): 547-64. https://doi.org/10.1007/s42010-019-00059-2.

Schiefner-Rohs, Mandy. 2018. «Mobile Learning und Schulentwicklung». In Handbuch Mobile Learning, herausgegeben von Claudia de Witt und Christina Gloerfeld, 637-56. Wiesbaden: Springer VS.

Stadler, Felix. 2016. Kultur der Digitalität. Erste Auflage, Originalausgabe. Berlin: Suhrkamp.

Stadler, Felix. 2018. «Herausforderungen der Digitalität jenseits der Technologie», Synergie 5: 8-16. https://uhh.de/kjeog. 